\title{
Nodule Deformation on Cleaning of PVA Roller Brushes and its Relation to Cross-contamination
}

\author{
Atsuki Hosaka ${ }^{1}$, Tsubasa Miyaki ${ }^{1}$, Yuki Mizushima ${ }^{2}$, Satomi Hamada ${ }^{3}$ \\ Ryota Koshino $^{3}$, Akira Fukunaga ${ }^{3}$, and Toshiyuki Sanada ${ }^{2 *}$ \\ ${ }^{1}$ Graduate School of Integrated Science and Technology, Shizuoka University, 3-5-1 \\ Johoku, Naka-ku, Hamamatsu, Shizuoka 432-8561, Japan \\ ${ }^{2}$ Department of Mechanical Engineering, Shizuoka University, 3-5-1 Johoku, Naka-ku, \\ Hamamatsu, Shizuoka 432-8561, Japan \\ ${ }^{3}$ Ebara Corporation, 4-2-1 Honfujisawa, Kanagawa, 251-8502, Japan \\ *sanada.toshiyuki@shizuoka.ac.jp
}

\begin{abstract}
This study investigates nodule deformation and contact area during PVA roller-type brush scrubbing to clarify their relationship with cross-contamination. Two high-speed video cameras with collimating LED light sources and an evanescent field on a prism enabled us to observe brush nodule deformation and contact area. Deformation analysis showed that the volume of a roller-type brush changes gradually at the beginning of compression, deforms more when vertically pushed at maximum compression, and then recovers rapidly at the end of compression. The brush contact area changes according to the brush and wafer rotation speed. The contact area can be categorized into three: the front, rear side face in the brush traveling direction, and vertically pushed bottom face on the surface. We analyzed the three types of brush contacts on a $100 \mathrm{~mm}$ type wafer and observed that the vertical compression type significantly affected the cross-contamination region.
\end{abstract}

Keywords: Cleaning, PVA brushes, Deformation, Cross-contamination, Nanoparticles

\section{Introduction}

Cleaning is a necessary process in semiconductor device manufacturing. In semiconductor cleaning, chemical and physical actions are used [1] owing to the need to remove ultrafine impurities in a limited time. A variety of physical cleaning methods are utilized including ultrasonic waves [2-4], droplet impingements [5-7], brush scrubbing [8-10], and lasers [11-13].

Brush scrubbing, a type of contact cleaning widely used for post-CMP cleaning, causes crosscontamination [14-15]. Contamination involves transferring the characteristic brush nodule shape patterns on a wafer after cleaning [16-17]. However, the effects of rotating both the brush and wafer have not been clarified. Besides, improving liquid mixing near the wall surface plays an important role in brush cleaning [18]. Nevertheless, the relationship between brush nodule deformation during scrubbing and liquid mixing has not been clarified.

In this study, we investigated the deformation and contact behavior of a roller-type PVA brush on a rotating wafer. We observed brush nodule deformation during rotation using two high-speed cameras and a collimated LED light source. We developed a device that reproduces the relative motion of the wafer and brush, and the brush contact was observed using the evanescent field generated on the prism. We analyzed the relationship between nodule deformation and cross-contamination patterns as reported in [16-17].

\section{Experimental}

Two experimental setups were used in this study. Fig. 1 shows a schematic of the experimental setup for observing the nodule deformation of a rollertype PVA brush during rotation and contact with a surface. The shape of the brush used is shown in the figure. A servo motor rotates the PVA roller brushes. A wide range of brush shapes can be obtained as the L-shaped gearbox changes the servomotor's rotation. The image was captured using two high-speed 
cameras and a collimating light source as a backlight. The brush nodule was compressed by 1 $\mathrm{mm}$ when it was located on the bottom surface. The brush rotation speed was set to $10 \mathrm{rpm}$. The volume change of the nodules was calculated from the reconstructed images by applying a series of image analyses obtained from the two directions. A frame rate of $300 \mathrm{fps}$ and a resolution of $1280 \times 800$ pixels of recording conditions were used.

Fig. 2 shows a method for observing the contact surface. This setup enabled us to distinguish the contact of the solid part of the PVA brush from the liquid or gas on the prism using the principle of total internal reflection. Details of this method are presented in Sanada et al. [19]. Furthermore, the relative motion of a roller brush rotating on the horizontal axis on a wafer $\left(\omega_{W}\right)$ rotating on the vertical axis $\left(\omega_{B}\right)$ was reproduced by two servomotors; see Miyaki et al. [18] for details. In this study, the relative behavior of all positions on a $100 \mathrm{~mm}$ wafer was visualized by changing the rotation speeds of the two motors and the contact positions of the brushes. A frame rate of 300 or 60 fps and a resolution of $1024 \times 1024$ or $2592 \times 2048$ pixels of recording conditions were used.

The obtained results were analyzed using image processing. Fig. 3 shows an example of an image captured in a nodule deformation experiment and the image processing results such as background processing, binarization, and size correction. Next, the volume change of the nodules was calculated. As shown in the figure, the nodule root was first detected. Then, the width of the nodules was calculated for the area below the line segment. Let these segments be $L_{x}$ and $L_{y}$ at different observation directions. The nodule widths $L_{x}$ and $L_{y}$ were calculated for each pixel up to the contact surface. In this experiment, the nodules do not cause excessive deformation as the rotation speed was as low as $10 \mathrm{rpm}$. Therefore, we assumed that the nodules maintained the shape of an ellipsoid, even though they were deformed. An ellipse was formed from the nodule widths $L_{x}$ and $L_{y}$ at each height, and they were stacked to reconstruct a solid. The volume was calculated by adding the areas of the ellipses and multiplying them by the conversion coefficient.

Fig. 4 shows an example of visualization of the contact surface. Background processing was performed after correcting the aspect ratio because the image was recorded using a tilted camera. An inversion processing was performed to emphasize the brightness.
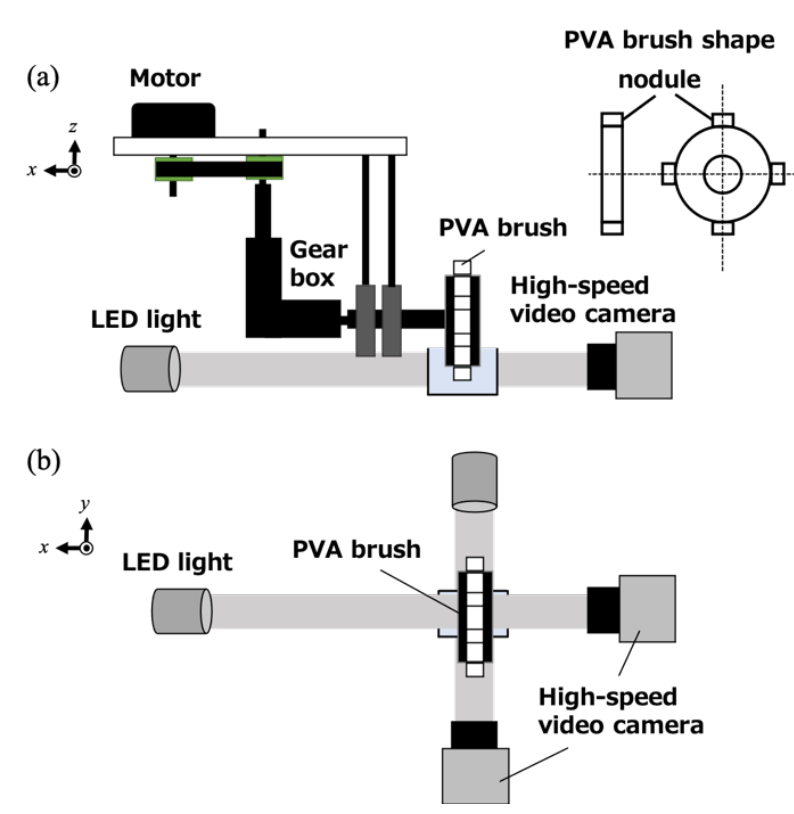

Fig. 1. Experimental setup for nodule deformation observation, (a) side view, (b) top view.

(a)

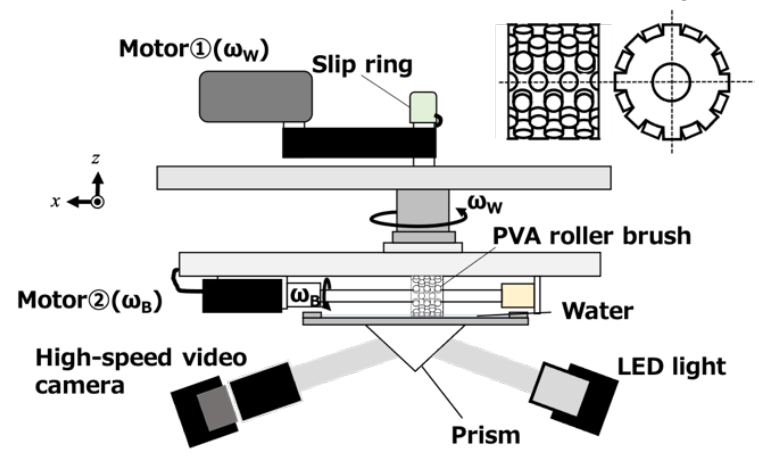

(b)

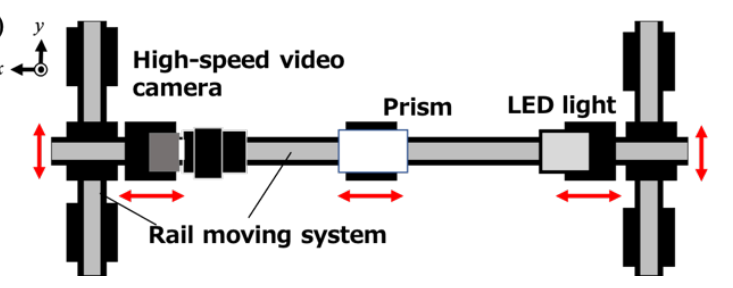

Fig. 2. Experimental setup for contact condition visualization, (a) side view, (b) top view. (a)

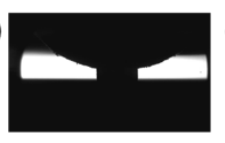

(d)

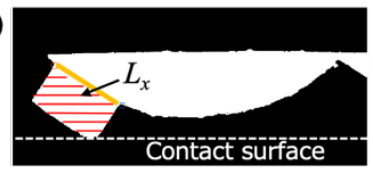

From $x$-direction

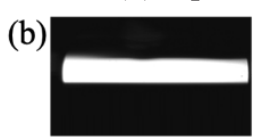

(c)
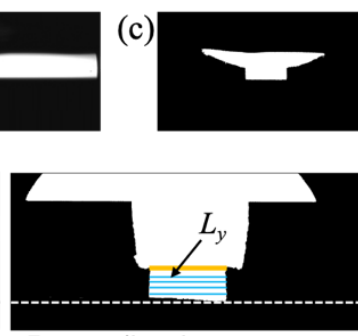

From $y$-direction
Fig. 3. Image processing for nodule deformation analysis, (a) original image, (b) background, (c) background processing and binarization, (d) volume change evaluation. 
(a)

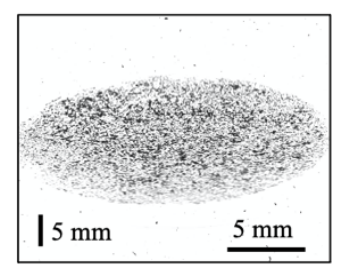

(b)

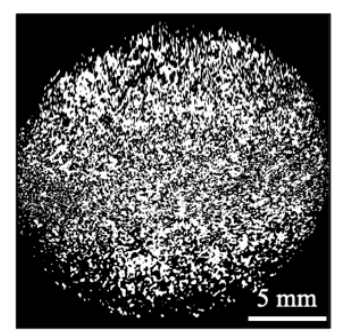

Fig. 4. Image processing for contact condition, (a) original image, (b) aspect ratio correction, binarization and black/white reversal.

\section{Results and discussion}

\subsection{Brush nodule deformation}

Fig. 5 shows an example of the nodule deformation of a PVA roller brush. Fig. 5 (a) shows an image taken from the $x$-direction and subjected to image processing. As shown in the figure, the brush makes contact with the edge, and the front side face of the nodule leading direction is pulled into the contact face. The shape of the brush quickly returns to its original form as the entrained surface recovers and separates from the surface. Fig. 5 (b) shows the three-dimensional reconstructed image of the nodules on the rotating brush at a given time. The result indicates that approximating the nodules to an ellipse to obtain the volume is a good approach. Fig. 5 (c) shows the change in the nodule volume per rotation. In the figure, the initial nodule volume of $98.96 \mathrm{~mm}^{3}$ and the volume of the nodules when vertically pushed to $1 \mathrm{~mm}$ at the location of the nodule on the bottom surface are indicated. We compared the interference volume, which defines interference with the contact surface when a rigid nodule is rotating.

Figure 5 (c) shows that the volume of the roller brush during sliding changes more than when vertically pushed in by $1 \mathrm{~mm}$. We considered that this is because the side face in the nodule leading direction is pulled into the contact surface, as shown in Fig 5 (a), resulting in a large volume change. Second, the volume change differs from the interference volume of the rigid nodule. The nodule volume gradually decreases at the beginning of compression, and recovers speedily at the end of compression. This is also related to the side face of the nodule. When the side face of the nodule is released from the contact surface, the nodule rapidly returns to its original shape, as shown in Fig. 5 (a). The volume change is different from the rigid nodule interference volume because of this motion. This result suggests that the volume of a normal brush nodule changes significantly each time it collides with a cleaning surface, and water retained in the brush desorb and adsorb each time. We considered that this water motion plays an important role in cleaning.

(a)

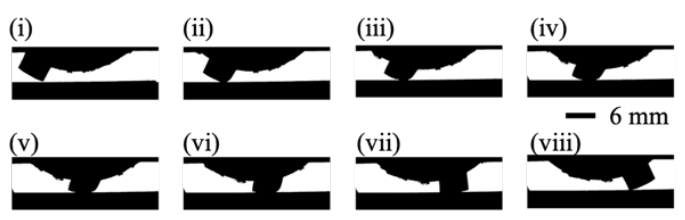

(b)
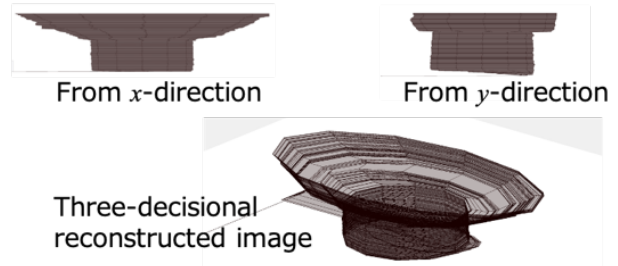

(c)

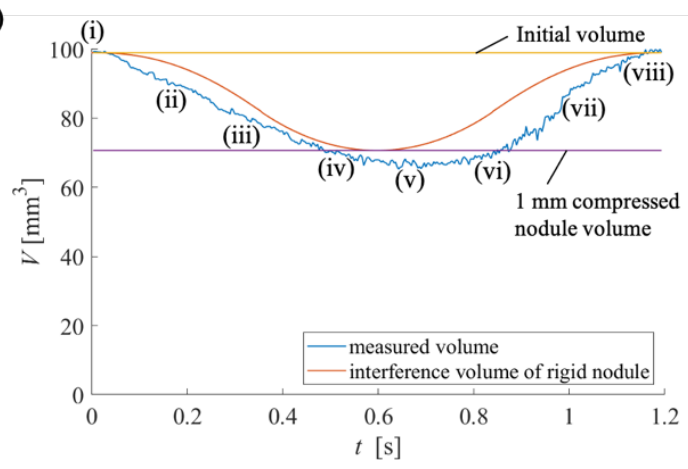

Fig. 5. Nodule deformation of a roller brush, (a) image sequence, (b) example of reconstructed image, (c) volume change.

\subsection{Contact shape}

The superimposed technique of 20 images was used to observe the path lines of the brush nodule contact. Figure 6 shows the path line of the three deformation patterns (Fg, Rg, and Pr type) defined by Miyaki et al. [18].

In the Fg type, which was observed in both large rotations of the brush and wafer, that is, a relatively large velocity condition, the sliding distance per nodule is small, limiting the line distance. In addition, the Fg type path line shows that the start and end of the nodule contacts are linear owing to deformation of the nodule side face, as discussed in the previous section. (a)

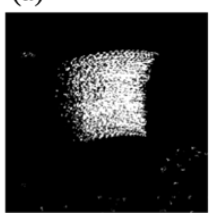

(b)

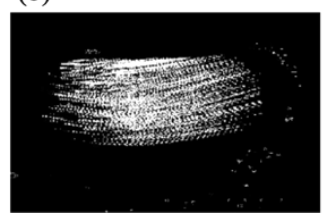

(c)

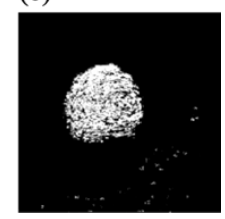

Fig. 6. Superimposed image of brush contact, (a) Front gap type (Fg), (b) Rear gap type (Rg), (c) Press type (Pr). 
(a)

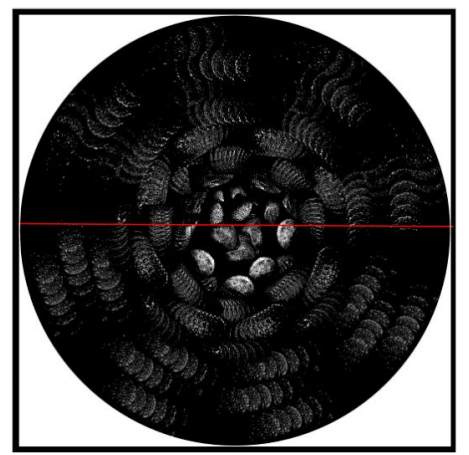

(c)

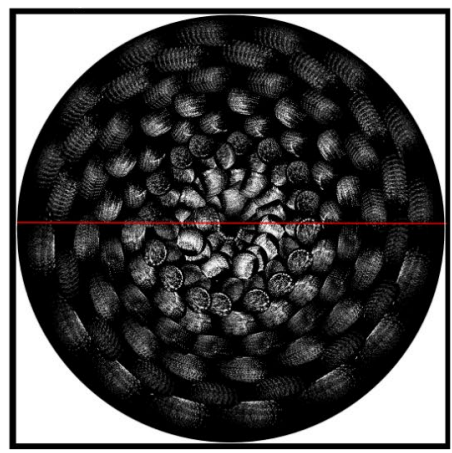

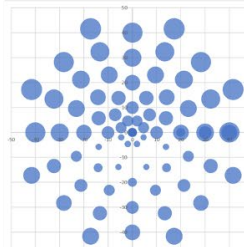

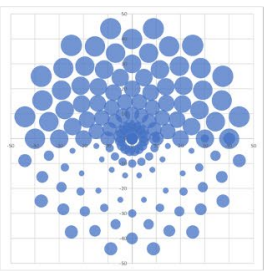

(b)
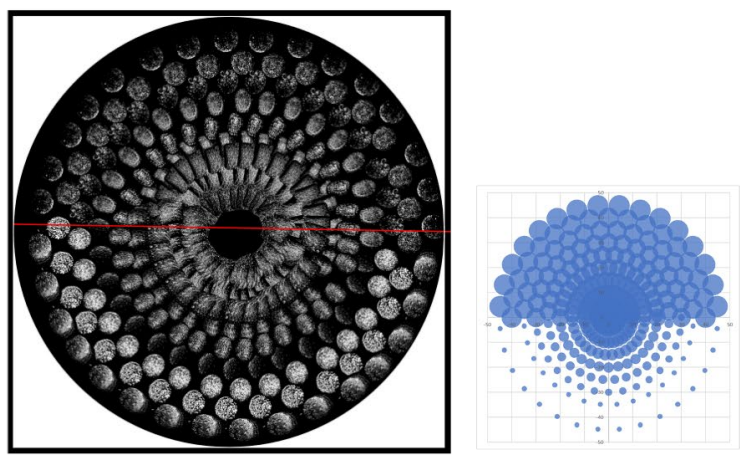

(d)
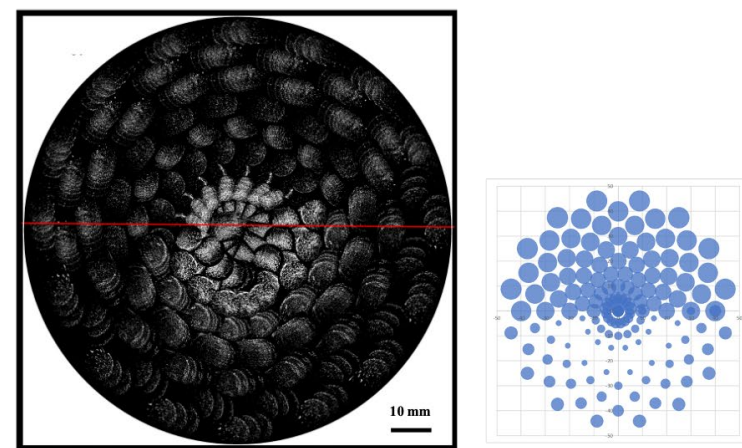

Fig. 7. Superimposed image of brush contact of 10 images on a $100 \mathrm{~mm}$ wafer until turning the half rotation and bubble chart for the relative velocity, (a) $\omega_{B}=25 \mathrm{rpm}, \omega_{W}=50 \mathrm{rpm}$, (b) $\omega_{B}=50 \mathrm{rpm}, \omega_{W}=25 \mathrm{rpm}$, (c) $\omega_{B}=25 \mathrm{rpm}$, $\omega_{W}=25 \mathrm{rpm},(\mathrm{d}) \omega_{B}=50 \mathrm{rpm}, \omega_{W}=50 \mathrm{rpm}$.

On the other hand, the sliding distance per nodule is large in the Rg type observed in the large wafer rotation and the same relative velocity direction, increasing the trajectory range. Furthermore, in the $\mathrm{Rg}$ type and deformation in which the nodules make smooth contact, the deformation is gradually pulled up while being relaxed. Therefore, both the start and end points of the nodule contacts have a semicircular shape. Finally, in the Pr type, one nodule is pushed in without sliding like a stamp so that its trajectory becomes circular. The nodule rotates during pushing and a clear path can be observed in the vertical compression type.

From our experience, a high cleaning performance can be generally achieved in the Fg type. Therefore, the Fg type, which compresses and slides simultaneously, plays an important role in cleaning compared with the Pr type, which only pushes vertically, and the Rg type, which has a long sliding distance. We considered that both deformation and sliding of the nodule are effective for mixing cleaning liquids.

\subsection{Contact map}

Fig. 7 shows the nodule contact path line shown in Fig. 6 at the contact position on a $100 \mathrm{~mm}$ wafer simulated by applying different wafer and brush rotations. It shows the nodule path lines of 10 images until turning the half wafer rotation, and the red line shows the starting point of the wafer rotation. The figure also shows the absolute value of the relative velocity between the nodule and the wafer in a bubble chart. The relative velocity is large in the upper half, and a negative relative velocity can be observed in the lower half. There is no relative velocity region in-between these two areas.

Figures 7 (a) and (c) show a comparison of the nodule contact conditions of the same brush rotation and different wafer rotation speeds. Because the rotation speed of the wafer in Fig. 7 (a) is larger than that in Fig. 7 (c), the angle of wafer rotation increases while a nodule collides with the next nodule so that the number of contacts made by the brush nodules decreases. Therefore, the sliding distance of one brush nodule becomes large, although there are many places where the PVA brush does not make contact with the wafer at half rotation. On the other hand, the positions where the relative velocity becomes zero in Fig. 7 (c) are far from the center of the wafer in Fig. 7 (a). The same trend is observed by comparing Figs. 7 (b) and (d). The zero relative velocity positions in Fig. 7 (b), which lower the wafer rotation, are separated from the center.

Next, we compared Figs. 7 (c) and (d), where the brush and wafer rotations are the same. There is no 
significant change in the relative speed, and the brush path pattern is similar. However, even if the speed ratio between the wafer rotation and the brush rotation is constant, the relative speed becomes zero positions in the radial direction changes depending on the absolute value of the speed. The position where the Pr type appears at zero relative velocity in Fig. 7 (c) is located on the outer side compared with that in Fig. 7 (d). In Fig. 7 (d), the maximum positive and negative relative velocities are located on the upper and lower semicircles so that the characteristic shapes in Fig. 6 clearly show these positions. Unfortunately, the contact path shape near the wafer center has a short sliding distance, which is similar to the Pr type path shape. Therefore, it is difficult to distinguish the $\mathrm{Fg}$ type or $\mathrm{Rg}$ type deformation model near the wafer center from the contact path. The original movie should be confirmed.

\subsection{Relation to cross-contamination}

Kim et al. [17] reported the distribution of residual particles due to cross-contamination on a wafer when the wafer rotation speed and brush gap are changed, that is, the amount of compression of a PVA roller brush. The authors reported the generation of a characteristic recontamination pattern that transfers brush nodule shapes. We compared this result with Fig. 7 and found that the contamination map and the Pr type position show good agreement. As shown in Fig. 5, the Pr type contact is pushed in like a stamp, resulting in twisting at that point [18]. Therefore, this result suggests that the Pr type nodule contact has a strong influence on cross-contamination. We considered that preventing Pr type contact by improving the nodule or brush shape or changing the rotation speed is important.

\section{Conclusion}

We observed the nodule deformation of rotating PVA brushes and the path of the contact point using high-speed photography. The nodule compresses gradually and immediately expands due to entrainment of the nodule side face during contact. We also showed that the vertically pushed type compression correlates with the location of crosscontamination. In the future, we plan to examine the relationship between nodule deformation and cleaning performance in detail.

\section{References}

1. T. Hattori, ECS Trans., 25 (2009) 3.

2. A. A. Busnaina, I. I. Kashkoush, and G. W. Gale,
J. Electrochem. Soc., 142 (1995) 2812.

3. W. Kim, T. H. Kim, J. Choi, and H. Y. Kim, Appl. Phys. Lett. 94 (2009) 081908.

4. S. Brems, M. Hauptmann, E. Camerotto, A. Pacco, A., T. G. Kim, X. Xu, K. Wostyn, P. Martens and S. De Gendt, ECS J. Solid State Sci. Technol., 3 (2013) N3010.

5. I. Kanno, N. Yokoi, and K. Sato, ECS Proc., 35 (1998) 54

6. H. F. Okorn-Schmidt, F. Holsteyns, A. Lippert, D. Mui, M. Kawaguchi, C. Lechner, P. E. Frommhold, T. Nowak, F. Reuter, M. B. Piqué, C. Cairós, and R. Mettin, ECS J. Solid State Sci. Technol. 3 (2014) N3069.

7. T. Sanada, and M. Watanabe, J. Photopolym. Sci. Technol., 28 (2015) 289.

8. A. A. Busnaina, H. Lin, N. Moumen, J. W. Feng, and J. Taylor, IEEE Trans. Semicond. Manuf., 15 (2002) 374 .

9. A. Philipossian and L. Mustapha, J. Electrochem. Soc., 151 (2004) G456.

10. K. Xu, R. Vos, G. Vereecke, G. Doumen, W. Fyen, P. W. Mertens, M. M. Heyns, C. Vinckier, and J. Fransaer, J. Vac. Sci. Technol., 22 (2004) 2844.

11. H. Horibe, T. Kamiura, K. Yoshida, J. Photopolym. Sci. Technol., 18 (2005) 181.

12. Hattori, T., ECS J. Solid State Sci. Technol., 3 (2013) N3054.

13. T. Kamimura, Y. Umeda, H. Kuramae, K Nuno, R. Nakamura, and H. Horibe, J. Photopolym. Sci. Technol., 31 (2018) 413.

14. J. H. Lee, M. Purushothaman, K. M. Han, H. Y. Ryu, N. P. Yerriboina, T. G. Kim, Y. Wada, S. Hamada, H, Hiyama, and J. G. Park, Polymer Testing, 77 (2019) 105921.

15. J. H. Lee, M. K. Poddar, K. M. Han, H. Y. Ryu, N. P. Yerriboina, T. G. Kim, Y. Wada, S. Hamada, H, Hiyama, and J. G. Park, Polymer Testing, 90 (2020) 106669.

16. K. Xu, R. Vos, G. Vereecke, G. Doumen, W. Fyen, P. W. Mertens, M. M. Heyns, C. Vinckier, J. Fransaer, and F. Kovacs, J. Vac. Sci. Technol., 23 (2005) 2160.

17. H. J. Kim, G. Bohra, H. Yang, S. G. Ahn, L. Qin, and D. Koli, Microelectron. Eng., 136 (2015) 36.

18. T. Miyaki, Y. Mizushima, S. Hamada, R. Koshino, A. Fukunaga, and T. Sanada, Solid State. Phenom., 314 (2021) 253.

19. T. Sanada, M. Hanai, A. Fukunaga, and H. Hiyama, Solid State. Phenom., 282 (2018) 73. 